\title{
HispanismeS
}

Revue de la Société des Hispanistes Français

15 | 2020

Laços de família de Clarice Lispector

\section{La rose parfaite}

La rosa perfecta

The Perfect Rose

Michel Riaudel

\section{CpenEdition}

\section{Journals}

Édition électronique

URL : https://journals.openedition.org/hispanismes/559

DOI : 10.4000/hispanismes.559

ISSN : 2270-0765

Éditeur

Société des Hispanistes Français

Référence électronique

Michel Riaudel, «La rose parfaite », HispanismeS [En ligne], 15 | 2020, mis en ligne le 01 juin 2020, consulté le 31 juillet 2021. URL : http://journals.openedition.org/hispanismes/559; DOI : https:// doi.org/10.4000/hispanismes.559

Ce document a été généré automatiquement le 31 juillet 2021.

\section{(2) $\odot \Theta \Theta$}

Les contenus de cette revue sont mis à disposition selon les termes de la Licence Creative Commons Attribution - Pas d'Utilisation Commerciale - Pas de Modification 4.0 International. 


\title{
La rose parfaite
}

\author{
La rosa perfecta \\ The Perfect Rose
}

Michel Riaudel

\section{Un drame domestique}

1 Les nouvelles de Clarice Lispector sont comme de petites pièces de théâtre, avec leur découpage en actes et en scènes, leurs décors, leurs personnages, leurs intrigues. «A imitação da rosa » se prête bien à cette façon d'organiser la lecture. Unité de lieu : un appartement du quartier de Tijuca, à l'époque généralement habité par une petite classe moyenne, en lisière de la zone nord de Rio. L'action va de la chambre (où se trouve la coiffeuse) à la cuisine (où Laura boit son verre de lait), puis de la cuisine au séjour dont l'élément central est le canapé. Concentration de temps : tout tient en une après-midi. Il faut dire qu'il ne se passe rien, ou presque, bien que ce soit la nouvelle la plus longue du recueil. Son étendue frappe d'autant plus qu'elle fait immédiatement suite à "Uma galinha", l'histoire la plus courte du livre. Un détail qui suggère l'attention qu'a portée l'écrivaine au rythme du recueil, au moment de son organisation.

2 On peut envisager divers découpages, comme par exemple deux actes et un épilogue. Acte 1 , scène 1 : Laura est perdue dans ses pensées; en attendant Armando, son époux, elle se prépare devant sa coiffeuse pour le dîner chez Carlota et João... Puis (scène 2), elle s'aperçoit qu'elle a oublié de prendre le verre de lait que lui a prescrit le médecin, ce qui l'amène d'abord à la cuisine, avant qu'elle ne s'installe sur le canapé du séjour, selon le rituel qu'elle a mis au point. Se déploie alors un autre moment indéterminé de longue rêverie, comme au début de la première nouvelle du recueil. Soudain (acte II), son regard est accroché par le vase de roses achetées le matin au marché. Désormais tout va tourner autour de ces fleurs. S'enchaînent une série de sentiments mêlés et d'envies contradictoires, d'hésitations. La bonne entre en scène, Maria a fini sa journée, Laura lui demande de faire un crochet, en rentrant chez elle, et d'apporter le bouquet à son amie Carlota. Épilogue : le soir est tombé (à Rio, il fait nuit vers 18-19 heures), le 
mari rentre du travail et trouve sa femme « sentada no sofá sem apoiar as costas, de novo alerta e tranquila como num trem. Que já partira. $»^{1}$ Autrement dit, la crise est revenue.

3 Car c'est là un autre prisme possible de lecture de ces treize nouvelles, qui s'organisent souvent autour d'un temps d'effondrement existentiel. Il peut être latent, comme au début de " Devaneio... », et bientôt accentué sous l'effet de l'alcool. Il peut survenir sans crier gare comme dans "Amor », et se creuser avec le passage par le Jardin des Plantes, à la fois inopiné et inéluctable comme les enchaînements implacables de la tragédie : si Ana ne rate pas l'arrêt où elle doit descendre, il n'y a plus d'égarement dans le monde fantastique du Jardim Botânico. Il peut précéder le début de l'histoire, comme dans « 0 búfalo ", d'où l'adversatif « Mas », très insolite en ouverture du récit...; la visite du zoo va néanmoins redéployer la crise en vagues successives, jusqu'à l'évanouissement conclusif. On sait qu'il y a crise quand le temps ordinaire est perturbé, quand tout s'intensifie, s'accélère, ou que tout est suspendu, que les personnages perdent leurs repères. Comme si l'on passait du chronos au kairos ou à l'aiôn, une temporalité à la fois contractée dans l'instant et dilatée dans l'éternel. Il faut, de ce point de vue, être très attentif au verbe "acontecer» (ou dans une moindre mesure «ocorrer»), qui fonctionne souvent comme un avertisseur.

4 Dans "A imitação da rosa ", " acontecer " revient quatre fois, au sein de contextes différents. Au deuxième paragraphe, il surgit au milieu d'une projection imaginaire, un retour au domicile familial après une escapade : «Como um gato que passou a noite fora e, como se nada tivesse acontecido, encontrasse sem uma palavra um pires de leite esperando » (p. 47-48, nous soulignons). Comme s'il ne s'était rien passé... Une forme de dénégation qui masque un "événement» dont on subodore, du fait du démenti, la gravité, recouverte toutefois d'une double couche de déréalisation : la métaphore et la condition. De façon générale, d'ailleurs, s'il se passe si peu de choses dans une nouvelle si longue, c'est que nous sommes transportés par le flux de conscience de Laura dans un temps parallèle, celui de la remémoration, de la méditation, des états d'âme, de la réflexivité... Le récit privilégie non pas l'action, mais la façon dont elle est vécue et ressentie, sur un mode subjectif. C'est au cours d'une de ces ruminations qu'est rappelé le conseil du médecin: "Abandone-se, tente tudo suavemente, não se esforce por conseguir - esqueça completamente o que aconteceu e tudo voltará com naturalidade" " (p. 50, nous soulignons).

5 Oublier ce qui s'est passé... Que s'est-il passé? Cela est suggéré plutôt que dit: un internement psychiatrique, qui a pris fin il y a peu. La crise aurait donc ici précédé le moment où le lecteur entre dans l'histoire. Dès les premières lignes, nous prenons en quelque sorte le train en marche - um trem que já partira - : c'est le moment du retour aux pénates, à la vie normale. Mais si nous devons deviner ce qui s'est passé (ce moment énigmatique ne va s'éclairer que progressivement, par bribes, à partir de plusieurs indices qui jalonnent le texte à cet effet : les précautions du mari, la réaction de l'entourage, l'infirmière, le médecin...), nous comprenons aussi que le rétablissement est fragile, qu'une nouvelle crise menace. Le lecteur est même en droit de se demander si Laura est vraiment "guérie", si la crise n'est pas chez elle structurante, en constante latence.

6 Troisième occurrence d'" acontecer ", dans l'épisode des roses : "Mas, sem saber por quê, estava um pouco constrangida, um pouco perturbada. Oh, nada demais, apenas acontecia que a beleza extrema incomodava » (p. 58, nous soulignons). La contemplation 
des roses trouble Laura, elle est dérangée par l'extrême beauté des fleurs. Ce n'est que cela, apenas, mais il n'empêche : quelque chose arrive à nouveau. C'est pourquoi, peut-être pour éviter que l'événement ne s'installe, elle imagine de faire porter le bouquet à son amie Carlota : donner les roses, c'était presque aussi beau que les roses elles-mêmes, penset-elle. Et elle ferait ainsi d'une pierre deux coups : « ela ficaria livre delas ». Mais alors, vient la question, le doute : « o que é mesmo que aconteceria »? (p. 59, nous soulignons). Dans ce cas, une fois délivrée des fleurs, qu'arriverait-il ? Cette récurrence du verbe " acontecer ", à quatre temps ou modes différents, rappelle à intervalles réguliers que la crise plane au-dessus du personnage, ne s'est résorbée qu'en surface, et qu'elle menace à tout moment de frapper à nouveau. Nous y reviendrons, car il faudra bien proposer des sens à cette « crise ».

7 Mais achevons le tracé de cette petite comédie avec la distribution des personnages. Comme nous l'avons vu, ils ne sont que trois à entrer en scène, une scène occupée sans interruption par la femme. Mais le récit, qui adopte presque constamment le point de vue de Laura en une focalisation interne dont le procédé domine le recueil, structure le drame et ses coulisses autour d'un quatuor, un carré parfait, traversé par des relations croisées : au parallélisme des deux couples se superpose un parallélisme des sexes, les hommes entre eux, les femmes entre elles, parlant de "choses de femmes ", un écho à Teresa Quadros (pseudonyme de Lispector) et à sa rubrique « Entre mulheres » (1952), ou bien à la chronique féminine de la supposée actrice Ilka Soares (autre avatar inventé par la journaliste Lispector en 1960, l'année où paraît Laços de família). L'univers de l'homme est ouvert sur l'extérieur, les grands espaces, il s'intéresse aux nouvelles du monde rapportées par les journaux; celui des femmes est cantonné aux affaires domestiques et à l'intime.

On sait peu de choses de João, une pièce marginale puisque la relation passe d'abord par une amitié d'enfance entre Laura et Carlota. Les épouses occupent de fait pour l'essentiel le premier plan du récit. Chacune incarne un profil de conduite et de conception de la femme qui doit inviter le lecteur à ne pas se contenter de schémas binaires (les dominants/les dominés...), ni réduire le recueil à un simple manifeste contre le patriarcat ou en faveur de l'émancipation des femmes. Certes, dans « la vraie vie », Clarice Lispector vient de laisser son mari en poste aux États-Unis et de rentrer à Rio avec ses deux fils quand elle publie Laços de família. Elle a connu le mariage et ses déboires, l'ennui d'une vie de femme de diplomate; elle découvre à partir de 1959 la condition de femme séparée (le divorce n'est officiellement reconnu au Brésil qu'en 1977, l'année de la disparition de l'écrivaine). Mais loin de se prêter à une lecture à sens unique, ses nouvelles sont attentives à toutes les nuances des codes genrés, qu'elles observent souvent du point de vue féminin, mais aussi parfois sous l'angle masculin, même quand le personnage central n'est pas un homme (voir la fin de "Os laços de família » ou de "A imitação da rosa ", précisément). Et surtout, elles juxtaposent des types de femmes très divers, de la vieille Anita de «Feliz aniversário ", accrochée à ses principes rigides et archaïques qui la font condamner presque la totalité de ses brus mais aussi de ses fils, à la jeune et plus indépendante Catarina ("Os laços de família ») ferraillant cordialement avec sa mère, la bien nommée Severina, en passant par la plus heureuse et la moins tourmentée d'entre elles, en dépit de toutes les contingences et adversités : « Petite fleur ». Ici Carlota incarne la vision " moderne », celle qui établit un rapport d'égalité dans le couple : "a amiga tinha um modo esquisito e engraçado de tratar o marido, oh não por ser "de igual para igual", pois isso agora se usava [...]» (p. 56). Ce qui frappe, dans le recueil, ce sont justement ces concomitances et concurrences 
de schémas opposant les générations entre elles, mais aussi des femmes de même âge, à un moment où, depuis les années 40, l'influence étasunienne de l'american way of life avec sa ménagère up to date, qui consomme, qui travaille, bouscule l'organisation traditionnelle de la famille. La prose de Lispector interroge, de ce point de vue, autant, sinon plus, la notion de norme, les rôles sociaux, les masques, que la "domination » elle-même. Les rapports de domination et de soumission traversent les classes et les sexes en tous sens.

9 L'amie, une femme audacieuse, effrontée, pour ne pas dire "libérée », est l'opposé de Laura, discrète, insipide, petite, sans grand charme. Depuis l'internat du Sacré Cœur où elles se sont connues, Carlota exerce une sorte de fascination sur sa camarade, prisonnière de l'image plus conventionnelle de l'épouse dévouée, dont la vie s'organise autour de celle du mari. Lui travaille, a une vie hors de la maison, tandis que Laura se doit d'être disponible quand il rentrera, d'être habillée, prête, à son service. Ses cheveux méticuleusement fixés par des épingles traduisent la contention de sa conduite. Sa tenue marron (qui réapparaît dans «O búfalo»), en harmonie avec la couleur de ses yeux et combinant avec la collerette crème en "dentelle véritable » ( $\mathrm{p}$. 49), habille un personnage effacé, le contraire de la femme volontaire qu'est Carlota, d'une bonté un tant soit peu autoritaire, nous dit le texte dans une de ces formules oxymoriques qu'affectionne Clarice Lispector. Ambitieuse et vive, individualiste, l'amie jouit de la vie, quitte à tricher, à mentir aux sœurs du pensionnat en prétendant avoir lu L'imitation de Jésus-Christ. Laura, elle, accorde du prix à l'authenticité : elle n'a pas les yeux verts des épouses qui cachent des choses à leur mari, ni la chevelure noire ou blonde des séductrices, mais les cheveux châtains. Elle est lente, soignée, sincère, appliquée ; le parangon du " deuxième sexe ", toujours au second plan et n'existant que pour mettre en valeur l'homme, aspirant même, avec l'amie comme avec l'époux, à un état d'insignifiance et d'oubli :

[...] recebendo enfim de novo a desatenção e o vago desprezo da amiga, a sua rudeza natural, e não mais aquele carinho perplexo e cheio de curiosidade - e vendo enfim Armando esquecido da própria mulher. E ela mesma, enfim, voltando à insignificância com reconhecimento. Como um gato que passou a noite fora e, como se nada tivesse acontecido, encontrasse sem uma palavra um pires de leite esperando (p. 47-48).

Comme s'il s'agissait de respecter les codes sans ostentation, d'atteindre une sorte de vérité du neutre, à mettre en parallèle avec l'aspiration pour le neutre de la narratrice de A paixão segundo G. H. : « Mas se eu cumprir meu núcleo neutro e vivo, então, dentro de minha espécie, estarei sendo especificamente humana $»^{2}$.

\section{Une femme « aliénée »?}

11 On aurait tôt fait de lire dans ce passage une confirmation de l'aliénation de Laura, si une analyse plus attentive n'amenait à nuancer la perspective. La question n'est en effet pas tant que Laura adopte une attitude soumise ("submissa»), mais qu'elle se détermine entre deux postures de l'entourage : ainsi, plutôt que la « tendresse perplexe et pleine de curiosité » que lui manifeste Carlota, elle préfère la "desatenção». Autrement dit, elle choisit volontairement de disparaître. De cette façon aussi, elle rejette le mensonge, les fausses attentions, ne veut pas être traitée comme une bête curieuse du fait de sa fragilité psychique. Car à défaut de la comprendre, on l'a enfermée - comme elle avait été enfermée «no tempo do Sacré Cœur ». L'histoire de 
Laura est à certains égards celle de la claustration. Sa "folie", traitée comme une maladie, médicalisée, l'a faite sortir du monde ordinaire. Or elle ne souhaite qu'une chose, revenir à cette banalité, pouvoir passer inaperçue, ne plus être pointée du doigt. Elle accepte qu'on l'aide, mais à certaines conditions :

As pessoas felizmente ajudavam a fazê-la sentir que agora estava "bem". Sem a fitarem, ajudavam-na ativamente a esquecer, fingindo elas próprias o esquecimento como se tivessem lido a mesma bula do mesmo vidro de remédio. Ou tinham esquecido realmente, quem sabe (p. 48).

L'important n'est pas qu'elle aille bien, mais qu'elle en ait ou donne le sentiment. Ce «bem » placé entre guillemets vient souligner qu'il n'est pas question d'une réalité vécue, mais d'un discours sur soi assumé par les autres et intégré par Laura elle-même. À l'inverse, trop d'attentions deviendrait suspect, peu naturel. Mieux vaut la rudesse de Carlota, sa vérité, car elle serait "naturelle »; de même les préconisations du médecin visent un retour au naturel: «tudo voltará com naturalidade» (p. 50); et Laura va s'asseoir avec son verre de lait sur le canapé, « "com muita naturalidade”, fingindo falta de interesse, "não se esforçando" » (p. 50), les guillemets venant à nouveau faire écran à cette prétendue spontanéité, ramener au discours (social) rapporté ce qui voudrait coller à l'authenticité de l'être. Puisqu'il faut jouer un rôle, autant adopter celui de l'effacement, couleur muraille. Être oubliée des autres, c'est s'assurer pour elle-même qu'elle est bien revenue dans le monde de la fatigue, de la banalité, de la normalité.

$\mathrm{Au}$ passage, on notera combien les métaphores sont faussement anodines et hasardeuses. Celle du retour du chat est doublement surprenante. Le " gato » est lourd de sous-entendus et d'associations en portugais: le terme désigne non seulement le félin, mais un bel homme, et suggère la virilité conquérante, avec ce que cela suppose de désir sexuel. Le lecteur peut s'étonner qu'il serve ici de comparant à Laura. Pourtant c'est bien elle qui a passé un temps hors de la maison, sans qu'il s'agisse de batifolages. Par ailleurs, la soucoupe de lait qui l'attend à son retour, négligemment posée à son attention, serait des plus ordinaires s'appliquant au chat, si elle n'appelait le prochain verre de lait : la boisson du bébé, la nourriture des premiers mois que pourvoit la mère (mais justement, de mère il n'y a point). En outre la blancheur laiteuse suggère volontiers des vertus apaisantes, une fonction de calmant, de médicament. Quant à la deuxième image, " como se tivessem lido a mesma bula do mesmo vidro de remédio " (p. 48), elle s'applique aux proches qui se rangent à la stratégie de Laura, l'oubli, se faire oublier... Or la comparaison rabat l'univers sémantique sur le champ médical : au lieu de conforter l'idée de guérison, l'image insiste sur le pathologique. On voit comment fonctionne le récit lispectorien, par approximations faussement inopinées, associations discrètes, secrètes, correspondances et paradoxes... Le travail critique consiste, entre autres, à dégager les effets de ces micro-télescopages.

14 Finalement, si Laura tient à être rassurée sur sa normalité, c'est qu'elle sait bien, au fond, au fond de son regard, qu'elle n'est en rien "normale». Sous trois aspects, au moins, signalés pour ainsi dire par symptômes : sa stérilité, son caractère maniaque, ses tendances surhumaines. D'abord, donc, derrière son air modeste, elle s'inquiète : «Por acaso alguém veria, naquela mínima ponta de surpresa que havia no fundo de seus olhos, alguém veria nesse mínimo ponto ofendido a falta dos filhos que ela nunca tivera?»(p. 48). Ce secret presque imperceptible, l'absence d'enfant, dirait son incomplétude, et expliquerait son instabilité ? La maternité l'aurait fait accéder à la plénitude de son sexe, alors qu'elle en est privée, qu'elle ne se sent femme qu'à moitié ? Cette thématique de la maternité, très présente chez Clarice Lispector, renvoie bien sûr 
à la «fonction femme » telle que la comprend la société, mais aussi au cycle spontané de la vie, à la perpétuation de l'espèce, tout comme au mystère de la création. Elle est la grande affaire de l'intime. Dans «Uma galinha ", la ponte de l'œuf fait voir la poule sous un autre jour et joue d'un effet de surprise: de l'intérieur inaccessible, énigmatique, imprévisible, du gallinacé, s'échappe un autre être en puissance (même si le texte corrige : en dépit des réflexes maternels de la poule, aucun poussin ne naîtra de l'œuf) ; dès lors la " maternité » la rend un temps intouchable, sacrée, l'élève au rang de « reine », comme dans les contes de fée. Au contraire, l'absence d'enfant de Laura la distingue d'Ana, de Catarina, de la rapariga... Elle est vécue comme une tare, comme une souffrance intime, de sorte que le personnage tient à l'expliquer «scientifiquement » : l'insuffisance ovarienne.

[...] de volta à paz noturna da Tijuca - não mais aquela luz cega das enfermeiras penteadas e alegres saindo para as folgas depois de tê-la lançado como a uma galinha indefesa no abismo da insulina -, de volta à paz noturna da Tijuca, de volta à sua verdadeira vida: ela iria de braço dado com Armando, andando devagar para o ponto do ônibus, com aquelas coxas baixas e grossas que a cinta empacotava numa só fazendo dela uma "senhora distinta"; mas quando, sem jeito, ela dizia a Armando que isso vinha de insuficiência ovariana, ele, que se sentia lisonjeado com as coxas de sua mulher, respondia com muita audácia: "De que me adiantava casar com uma bailarina?", era isso o que ele respondia (p. 54-55).

Le passage appelle plusieurs remarques. Incidemment, Laura est assimilée à une poule sans défense quand, à l'hôpital, les infirmières la plongent dans un coma insulinique (un traitement de choc alors en vogue, supposé guérir de la schizophrénie, mais en réalité inefficace et aux effets secondaires dévastateurs). Nul besoin d'insister sur l'interpolation avec la volaille de la nouvelle qui précède dans le recueil. Soulignons en revanche le "senhora distinta ", ce statut à part (distinct, mais aussi distingué pour qui veut l'entendre ainsi) qui tient à son infécondité comme à son corps disproportionné, loin des canons du papier glacé des magazines : des cuisses courtes et grasses, mises en relief par une ceinture serrée (voir le disgracieux « empacotava »). Pour la flatter ou parce qu'il affectionne sincèrement un autre idéal féminin que le corps gracile et élancé de la ballerine (à nouveau il faut entendre dans la réplique du mari, répétée à l'envi, un sens à tiroirs : l'archétype de la beauté, mais aussi ce côté cocotte ou prostituée que le monde bourgeois associe à la danseuse, illustré entre autres par Nana), Armando la rassure en opposant deux systèmes de valeurs, le leur, à usage interne, et celui du monde environnant au jugement vain et superficiel. Enfin, ce passage développe une projection, une nouvelle rêverie de Laura, dans laquelle elle se met en scène dans la rue, sous le regard de tous, au bras de son époux. C'est bien là une des dimensions " aliénées » de la vie de Laura, telle qu'elle cherche à correspondre, avec ses moyens, aux attentes d'autrui. Aliénée non pas au sens psychiatrique de folle, ou au sens marxiste de soumise à l'idéologie dominante, mais en ce qu'elle est jusqu'à un certain point étrangère à elle-même, soit par les « discours » qu'elle endosse, soit par les rôles qu'elle accepte de jouer. Pour normaliser sa condition stérile, elle la médicalise (c'est-àdire qu'elle reprend à son compte le discours de l'autre, de la science, du médecin) : une façon de se dédouaner, de ne pas vivre son incomplétude comme un manque, une faute, de l'ôter du domaine moral ou psychologique pour l'ancrer dans l'ordre physiologique. De la sorte, elle dissocie son être profond, ses ressorts les plus enfouis, et celui qu'elle essaie de faire coïncider avec certains codes en vigueur. Il y a chez elle du calcul, une adhésion de surface au stéréotype. Ainsi opère le présent de vérité générale de « La paix d'un homme est... », où la valeur proverbiale énonce une sorte de loi de la nature. 
De même, au Sacré Cœur, elle recopiait les cours à la perfection, mais sans les comprendre; de même elle lisait "avec l'ardeur d'un âne» le livre de piété, en pressentant toutefois le danger qu'il y avait pour elle à s'y abandonner vraiment. Étrangère, elle l'est dans sa propre maison qu'elle voit comme une salle d'attente : «Oh como era bom rever tudo arrumado e sem poeira, tudo limpo pelas suas próprias mãos destras, e tão silencioso, e com um jarro de flores, como uma sala de espera. Sempre achara lindo uma sala de espera, tão respeitoso, tão impessoal.» (p. 57).

L'autre signe d'un grain de sable venant gripper cette existence ordinaire tient au comportement obsessionnel ou maniaque de Laura, «seu gosto minucioso pelo método » (p. 48) pour le dire positivement. Elle range la maison, repasse..., tout est prétexte à mise en ordre. Ce que mimétise le récit par des répétitions abondantes, qu'on mettra en relation avec le commentaire de Clarice Lispector sur l'écriture de la nouvelle :

"Imitação" me deu a chance de usar um tom monótono que me satisfaz muito: a repetição me é agradável, e repetição acontecendo no mesmo lugar termina cavando pouco a pouco, cantilena enjoada diz alguma coisa ${ }^{3}$.

17 À la redondance s'ajoute cette façon insolite de structurer le texte à partir de listes : «[...] ela não precisasse fazer mais nada, senão $1^{\circ}$ ) calmamente vestir-se; $\left.2^{\circ}\right)$ esperar Armando já pronta; $3^{\circ}$ ) o terceiro o que era? Pois é. Era isso mesmo o que faria. » (p. 48). Ce souci du rangement, de la propreté, de l'hygiène, remonte à l'enfance, nous est-il dit. Mais il tourne à l'obsession qui ne va pas sans une certaine vacuité: un «troisièmement » sans objet, un propos qui tourne en rond, mais a l'avantage de ritualiser la routine et de remplir le vide d'une « intime richesse »...

O que devia fazer, mexendo-se com familiaridade naquela íntima riqueza da rotina - e magoava-a que Carlota desprezasse seu gosto pela rotina - o que devia fazer era $1^{\circ}$ ) esperar que a empregada estivesse pronta; $2^{\circ}$ ) dar-lhe o dinheiro para ela já trazer a carne de manhã, chã de dentro; como explicar que a dificuldade de achar carne boa era até um assunto bom, mas se Carlota soubesse a desprezaria; $3^{\circ}$ ) começar minuciosamente a se lavar e a se vestir, entregando-se sem reserva ao prazer de fazer o tempo render (p. 54).

De nouveau, on ne psychiatrisera pas outre mesure ce qui peut s'apparenter à des troubles obsessionnels de comportement, mais il est plus intéressant de relever que, comme ailleurs dans le recueil, la focalisation interne et le recours à l'indirect libre ont pour effet fusionnel de contaminer le récit hétérodiégétique, de lui donner les couleurs de la façon de parler et de penser du personnage. Aliénée, elle l'est donc également, ici, au sens où le rôle qu'elle joue la tient à distance de sa vérité intrinsèque autant qu'il signalerait une difficulté à s'inscrire dans la réalité. On l'a déjà vu dans la manière dont le récit s'étoffe autour de petits riens: les maigres résurgences du présent sont entrecoupées de longues considérations nébuleuses, fantasmatiques ou irréelles, allant et venant dans des strates de passé et de futur plus ou moins proche (pour une part, les coulisses du théâtre, le hors champ qui a, du point de vue narratif, l'avantage de donner une épaisseur diachronique au personnage), de conditionnels et de subjonctifs. Témoin de cette perte de contact avec le réel, cette alerte : «Mas, quando viu as horas... » (p. 49), qui aura la vertu de relancer l'«action». Plus loin, elle s'assoit sur son canapé comme si elle se rendait visite à elle-même, dans une forme de dédoublement: «Sentou-se no sofá como se fosse uma visita na sua própria casa que, tão recentemente recuperada, arrumada e fria, lembrava a tranquilidade de uma casa alheia » (p. 50). 
L'autre extraordinaire exemple de cette dissociation est fourni par le paragraphe martien :

Se uma pessoa perfeita do planeta Marte descesse e soubesse que as pessoas da Terra se cansavam e envelheciam, teria pena e espanto. Sem entender jamais o que havia de bom em ser gente, em sentir-se cansada, em diariamente falir; só os iniciados compreenderiam essa nuance de vício e esse refinamento de vida (p. 51). écho, pour le lire de façon triviale, à l'aventure spatiale déclenchée à l'après-guerre (et, de façon générale, à l'attention de Clarice Lispector pour les sciences, le monde de l'atome, des "partículas », p. 53), mais il illustre surtout combien Laura vit sur une autre planète et combien elle observe et rêvasse plus qu'elle ne vit (à la différence de la très pratique et pragmatique Carlota). La coiffeuse, meuble féminin par excellence, dédié à l'apprêt, la mise en scène du corps féminin - le paraître maquillant l'être - est aussi propice à la mise à distance de soi et à l'introspection. Devant la glace, la femme s'évalue, se demande qui elle est... À vrai dire, le meuble a surtout cette fois une fonction narrative: introduire la description physique du personnage à travers sa propre vision ${ }^{4}$; et il nous montre par ailleurs une Laura à nouveau occupée à ordonner, ranger (et parfois à planifier de le faire).

Mars, miroir aux vertus plus grossissantes, nous introduit lui au troisième "symptôme ", celui pour lequel tout indique que Laura a été internée : le syndrome de la perfection. L'humanité, regardée de Mars, se définit par sa faillibilité, sa fatigue. Or Laura ne dormait plus. Et la sensation qui la fait renouer avec l'épuisement la rassure sur son état : " no cansaço havia um lugar bom para ela, o lugar discreto e apagado de onde, com tanto constrangimento para si e para os outros, saíra uma vez. Mas como ia dizendo, graças a Deus, voltara.» (p. 53). De même qu'elle fait durer le temps inutile, les courses au marché..., elle étire sa réflexion sur la lassitude, son envie de dormir, sa tentation de faire une sieste :

Mas não tinha verdadeiramente tempo de dormir agora, nem sequer de tirar um cochilo - pensou vaidosa e com falsa modéstia, ela era uma pessoa tão ocupada! sempre invejara as pessoas que diziam "não tive tempo" e agora ela era de novo uma pessoa tão ocupada (p. 53-54).

Le rôle endossé par Laura consiste, et c'est là toute sa contradiction, à redevenir normale, pareille aux autres à la perfection.

\section{Imitation, tentation, perfection}

Il est temps de faire un sort à la mention de L'imitation de Jésus-Christ, ouvrage auquel fait écho le titre de la nouvelle, et qui apparaît explicitement à deux reprises dans le récit. La première occurrence nous ramène au temps du Sacré Cœur.

Quando lhe haviam dado para ler a "Imitação de Cristo", com um ardor de burra ela lera sem entender mas, que Deus a perdoasse, ela sentira que quem imitasse Cristo estaria perdido - perdido na luz, mas perigosamente perdido. Cristo era a pior tentação. E Carlota nem ao menos quisera ler, mentira para a freira dizendo que tinha lido (p. 49).

$23 \mathrm{Au}$ contraire de Carlota, Laura s'est efforcée de lire ce manuel ascétique de la fin du Moyen Âge, comme lui demandaient les sœurs du pensionnat mais, tout en disant ne rien y comprendre (à moins que ce ne soit le commentaire du narrateur), elle en tire des conclusions inverses au propos de l'ouvrage, conclusions qui ne sont pas loin de 
rappeler les notes de l'auteure, puisque Clarice Lispector est revenue elle-même à plusieurs reprises sur ce classique. Le 14 août 1946, alors qu'elle vit à Berne, elle en parle en ces termes à sa sœur Tania :

Ah, querida, como estou cansada de ter saudade e de pensar. Estou tão cansada disso e de tentar pelo pensamento sair fora da vida que levo que não tenho gosto nem força de trabalhar. Um dia desses abri um livro que comprei, o célebre Imitação de Cristo, e estava escrito: ainda não sofreste até o sangue. Acho que no momento em que isto suceder, será o momento de agir, de resolver. Por enquanto ainda posso contemporizar ${ }^{5}$.

Le même jour, elle écrit à son ami et jeune écrivain Fernando Sabino :

Quanto à Imitação de Cristo, ela manda sofrer até o sangue, e me ceder inteiramente. Sofrer até o sangue, chegarei lá e mesmo às vezes já cheguei. Mas me abandonar, não sei como, me falta a graça. Como diz Álvaro Lins, eu sou dos muitos chamados e não escolhidos... ${ }^{6}$

Le 5 octobre 1953, soit sept ans plus tard, elle revient avec lui sur le sujet :

Quanto às leituras, variadas, provavelmente erradas, a mais certa é a Imitação de Cristo, mas é muito difícil imitá-LO, e isso é menos óbvio do que parece ${ }^{7}$.

On voit que le livre a marqué Lispector de façon durable. Il s'instaure dans sa lecture une sorte de relation sadomasochiste, qui ne l'effraie pas, bien qu'elle ait ses limites. La demande d'imitation lui semble très exigeante, au-delà du possible. Sans bien sûr superposer l'écrivaine et son personnage, on constate qu'elle transpose dans la nouvelle une sorte de relation fascinée où Laura touche elle aussi ses propres limites : d'entendement et de sacrifice. Mieux, la fiction glisse de l'imitation à la tentation, dans une transformation paradoxale du Christ en tentateur: inversion des épreuves qu'il aurait subies dans le désert. Dieu et diable se rapprochent.

[...] quem imitasse Cristo estaria perdido - perdido na luz, mas perigosamente perdido. Cristo era a pior tentação (p. 49).

Imiter, c'est risquer de se perdre dans la lumière, s'exposer à un danger. Il y a une séduction périlleuse de l'extrême, de la radicalité de l'épreuve, à laquelle résiste finalement Laura. Au lieu du salut, c'est la perdition qui attend celui qui s'aventurerait à imiter le Christ. Lumière et perdition... Le Christ était la pire tentation. Clarice Lispector prend un malin plaisir à cultiver les contradictions et à retourner les signes. Ce glissement qui mène de l'imitation à la tentation précède pas moins de six autres retours du sème, riches en significations.

En premier lieu la tentation se rapporte à la période où elle devient surhumaine, autrement dit celle qui a précipité son internement et qu'elle veut écarter avec force : "Oh, fora apenas uma fraqueza; o gênio era a pior tentação ". Il faut résister à la perfection du surhomme, comme on doit résister à l'imitation du Christ. Plus loin, Laura repousse la tentation de faire un somme. Paradoxalement, elle choisit l'ascèse qu'elle avait rejetée. Mais bientôt ce sont les roses qui se mettent sur son chemin, tel un démon séducteur: "Como uma viciada, ela olhava ligeiramente ávida a perfeição tentadora das rosas, com a boca um pouco seca olhava-as ». On appréciera le parcours sémantique, de l'imitation à la tentation, puis de la tentation à la perfection. Citons in extenso le dernier paragraphe où figure le verbe « tentar » :

Quando Maria voltou e pegou o ramo, por um mínimo instante de avareza Laura encolheu a mão retendo as rosas um segundo mais consigo - elas são lindas e são minhas, é a primeira coisa linda e minha! e foi o homem que insistiu, não fui eu que procurei! foi o destino quem quis! oh só dessa vez! só essa vez e juro que nunca mais! (Ela poderia pelo menos tirar para si uma rosa, nada mais que isso: uma rosa 
para si. E só ela saberia, e depois nunca mais oh, ela se prometia que nunca mais se deixaria tentar pela perfeição, nunca mais!) (p. 64). de garder les roses pour elle et la résolution de les donner à son amie. Les exclamations signalent l'ardente passion qui plaide pour conserver le bouquet. La parenthèse abrite un argument inavouable, comme si ce qui y était dit pouvait échapper à Dieu qui voit et entend tout (mais pas ce qui est entre parenthèses). Il s'agit d'ailleurs d'y tricher avec la réalité : faire porter les fleurs, mais en distraire une pour un «bonheur clandestin » titre d'une nouvelle et d'un recueil paru en 1971 avec quelques inédits. Dans tous les cas, garder les roses apparaît comme une hypothèse transgressive, une possibilité exceptionnelle à ne jamais répéter. En sa faveur, parmi les raisons invoquées, il y a celle de la possession : ce serait la première fois que Laura aurait quelque chose de joli à elle. Posséder, c'est aussi comme s'appartenir, elle qui est jusque-là passée des mains de son père et du prêtre à celle de son mari : " ele que a recebera de um pai e de um padre " (p. 52).

Néanmoins l'alibi du destin repose à nouveau sur la tentation d'un homme, le fleuriste qui a insisté pour qu'elle achète ses roses. Un homme de plus sur son chemin ? Il y a en fait une grande différence entre son mari et ce vendeur enjôleur, suborneur (auquel elle a cette fois succombé). Armando est timide ; quand il va la voir à l'hôpital, il ne sait que faire d'elle. Si Laura avance un commentaire sur la conduite de son amie, il concorde comme pour éluder la discussion : « Armando concordara mas não dera muita importância » (p. 56). Le fait qu'il se satisfasse d'une épouse petite, trapue, en lui opposant la danseuse, s'inscrit de même dans cette physiologie du mariage qui ne permet, selon les consignes du "prêtre austère ", que "a alegria humilde e não a imitação de Cristo » (p. 67). Formule décisive, qui oppose précisément le train-train ordinaire et sans joie cultivé par un mari faible, veule, qui s'emploie à occulter les aspirations de sa femme pour la "perfection", et d'autre part les "tentations » de Laura. Tentation mystique (le livre de piété), tentation esthétique (les fleurs) et tentation de l'extase physique. En effet elle oscille en permanence entre le jeu de rôles et l'envie de s'abandonner, de jouir corps et âme. Imiter le Christ, imiter la rose, accepter le "bliss ${ }^{8}$ ", ce serait en quelque sorte se laisser dévorer, engloutir. Voilà pourquoi l'imitation est violente, dangereuse et pour ainsi dire impossible ou interdite : parce que Laura ne peut imiter, elle ne peut que devenir ce qu'elle imite, se transformer, passer de l'autre côté du miroir, se métamorphoser comme la fin le suggère.

[Armando] Abriu a boca e involuntariamente a cara tomou por um instante a expressão de desprendimento cômico que ele usara para esconder o vexame quando pedira aumento ao chefe. No instante seguinte, desviou os olhos com vergonha pelo despudor de sua mulher que, desabrochada e serena, ali estava (p. 69).

Le participe "desabrochada" se déplace du vocabulaire des fleurs à la femme, épanouie, éclose, que le mari ne peut regarder. Et si la «folie " n'était pas celle de Laura, mais de cet époux et de cet entourage qui ne supportent pas, n'autorisent pas le ravissement féminin, indécent, impudique, la jouissance religieuse, artistique, charnelle?

Ce finale appelle plusieurs remarques, la première étant que le point de vue s'est déplacé : la scène est vue à travers les yeux d'Armando, recours narratif suffisamment extraordinaire pour être souligné. Bien que Laura suggère elle-même le retour de «sa folie » et l'annonce à son mari, la question est bien située dans la manière dont il la 
regarde et s'interdit de la voir, comme endossant le commentaire naguère assumé par Laura: "o principal nunca fora a beleza» (p.50). Elle, en revanche, a cédé à la tentation, à la perfection, elle est devenue littéralement rose (la rose, qui incarne traditionnellement la beauté féminine). À la fois assise et en mouvement, emportée, transportée. Incapable d'embarquer avec elle, l'époux reste à quai. Les craquelures ont changé de camp. Un peu plus haut, la lucidité aussi est passée du côté de Laura, avec quelques latences érotiques lorsqu'elle imagine l'arrivée d'Armando :

Armando abriria a porta. Apertaria o botão de luz. E de súbito no enquadramento da porta se desnudaria aquele rosto expectante que ele procurava disfarçar mas não podia conter. Depois sua respiração suspensa se transformaria enfim num sorriso de grande desopressão. Aquele sorriso embaraçado de alívio que ele nunca suspeitara que ela percebia. Aquele alívio que provavelmente, com uma palmada nas costas, tinham aconselhado seu pobre marido a ocultar (p. 66-67, nous soulignons).

Au tour d'Armando de feindre, de masquer de profondes failles, comme cela lui a été conseillé. À fêlure, fêlure et demie, chacun évoluant au-dessus d'un volcan, d'une béance sismique. Si Laura peut, pour sa part, annoncer que « c'est revenu », c'est qu'elle a lâché prise. Le «desabrochada » est de ce point de vue bien plus qu'une métaphore, comme nous le suggérons, car la femme s'est incarnée en bouquet, ce que la prose lispectorienne permet et pratique en abondance. Il y a en effet un rapport phénoménologique aux choses, qui abolit la coupure sujet/objet (d'où la fréquence de la focalisation interne et de l'indirect libre ${ }^{9}$ ), et tend à estomper les frontières (intérieur/extérieur etc.); d'où aussi la limite ténue entre métaphore et métamorphose, et entre imitation et authenticité : « Noto que os meus imitadores são melhores que eu. A imitação é mais requintada que a autenticidade em estado bruto ${ }^{10}$ ».

Concluons en revenant au paragraphe de l'«explication qui n'explique pas » ou " explication inutile " sur la composition de "A imitação da rosa ", dont nous n'avons cité que les dernières phrases. Ainsi commence-t-elle :

"Imitação da rosa" usou vários pais e mães para nascer. Houve o choque inicial da notícia de alguém que adoecera, sem eu entender por quê. Houve nesse mesmo dia rosas que me mandaram, e que reparti com uma amiga. Houve essa constante na vida de todos, que é a rosa como flor. E houve tudo o mais que não sei, e que é o caldo de cultura de qualquer história ${ }^{11}$.

Il y a toujours comme un nouveau roman dans ces exégèses fournies par l'écrivaine, une métafiction pour une part mimétique de la nouvelle concernée, pour une part nouvelle bifurcation. Ici l'histoire serait née de plusieurs parents comme Laura est passée d'homme en homme. Et elle serait la concrétion d'une série d'anecdotes contingentes et d'un bouillon de culture. Clarice Lispector n'y dit rien de ses tentatives de lecture de l'Imitation de Jésus-Christ. La genèse garde ses secrets.

\section{BIBLIOGRAPHIE}

Odile CISNEROS, « O último sopro de Clarice: Um sopro de vida como Ars Poetica », Revista da Anpoll, vol. 51, n. esp., Florianópolis, (janv-déc. 2020), p. 83-94. 
Roberto CORRÊA DOS SANTOS, Clarice, ela, São Paulo, Instituto Moreira Salles, 2012.

Alex KEINE DE ALMEIDA SEBASTIÃo, « O manuscrito de Um sopro de vida: imagens da letra », Vertentes \& Interfaces I: Estudos Literários e Comparados, Vitória da Conquista, vol. 12, n², (juil.-déc. 2020), p. 19-38.

Clarice LISPECTOR, A paixão segundo G. H., Rio de Janeiro, Editora do Autor, 1964.

Clarice LISPECTOR, Laços de família [1960], Rio de Janeiro, Francisco Alves Editora, 1990, $20^{\mathrm{e}}$ éd.

Clarice LISPECTOR, Um sopro de vida, Rio de Janeiro, Círculo do livro, 1978.

Clarice LISPECTOR, A descoberta do mundo, Rio de Janeiro, Nova Fronteira, 1984.

Clarice LISPECTOR, Fernando SABINO, Cartas perto do coração, Rio de Janeiro, Record, 2002.

Clarice LISPECTOR, Minhas queridas, Rio de Janeiro, Rocco, 2007.

Carlos MENDES DE SOUSA, « Sete semanas ou o livro por vir: Um sopro de vida » [colloque international en ligne], 100 anos de Clarice Lispector, table 5 (20 octobre 2020) [disponible le 08/03/21], <URL : https://www.youtube.com/watch?v=4bCo4IREjBM\&t=8s>

\section{NOTES}

1. Clarice LISPECTOR, Laços de família, Rio de Janeiro, Francisco Alves Editora, 1990, p. 69. Les citations de la nouvelle analysée seront désormais indiquées par le numéro de la page entre parenthèses.

2. Clarice LISPECTOR, A paixão segundo G. H., Rio de Janeiro, Editora do Autor, 1964, p. 125.

3. Clarice lisPeCtoR, A descoberta do mundo, Rio de Janeiro, Nova Fronteira, 1984, p. 365. Rappelons que « A explicação que não explica », où l'écrivaine commente la genèse des nouvelles de Laços de familia, paraît d'abord dans la presse, puis intègre sous le titre «A explicação inútil » la section «Fundo de gaveta » de la première édition de A legião estrangeira, en 1964 (section qui disparaît dans les éditions suivantes, et sera rééditée séparément sous le titre Para não esquecer) ; le texte est ensuite repris sous ce nouveau titre dans une chronique du Jornal do Brasil, à la date du 11 octobre 1969, est incorporé à l'édition posthume de A descoberta do mundo.

4. Roberto Corrêa dos Santos voit ici une "fausse troisième personne", ce qui revient à confondre récit à la troisième personne et récit à la première personne, alors que c'est précisément ce jeu indéterminé et subtil qui constitue l'une des forces de ce texte. Cf. Roberto CORRÊA DOS SANTOS, Clarice, ela, São Paulo, Instituto Moreira Salles, 2012, p. 37.

5. Clarice LISPECTOR, Minhas queridas, Rio de Janeiro, Rocco, 2007, p. 154.

6. Clarice LISPECTOR ; Fernando SABINO, Cartas perto do coração, Rio de Janeiro, Record, 2002, p. 51.

7. Ibid., p. 112.

8. En référence à la nouvelle « Bliss » de Katherine Mansfield, où le poirier du jardin occupe d'une certaine façon pour Bertha la place du bouquet de roses pour Laura.

9. Et les flottements entre la figure de l'écrivaine et ses personnages, dans A hora da estrela et Um sopro de vida.

10. Nous prenons la liberté de citer Um sopro de vida (Rio de Janeiro, Círculo do livro, 1978, p. 35), en dépit des problèmes génétiques qui pèsent sur ce livre posthume dont l'édition a été assurée par Olga Borelli : voir par exemple Odile CISNEROS, «O último sopro de Clarice: Um sopro de vida como Ars Poetica », Revista da Anpoll, vol. 51, n. esp., Florianópolis, (janv-déc. 2020), p. 83-94 ; Alex KEINE DE ALMEIDA SEBASTIÃo, "O manuscrito de Um sopro de vida: imagens da letra », Vertentes \& Interfaces I: Estudos Literários e Comparados, Vitória da Conquista, vol. 12, n² (juil.-déc. 2020), p. 
19-38; et la conférence de Carlos MENDES DE SOUSA, « Sete semanas ou o livro por vir : Um sopro de vida» [colloque international en ligne], 100 anos de Clarice Lispector, table 5 (20 octobre 2020) [disponible le 08/03/21] <URL : https://www.youtube.com/watch? $\mathrm{v}=4 \mathrm{bCo} 4 \mathrm{IREjBM} \& \mathrm{t}=8 \mathrm{~s}>$

11. Clarice LISPECTOR, A descoberta do mundo, op. cit., p. 365.

\section{RÉSUMÉS}

Aussi longue qu'énigmatique, la nouvelle " $\mathrm{A}$ imitação da rosa ", qui met en scène deux modèles féminins, Laura et son amie Carlota, semble labyrinthique. Autour des relations des deux femmes qui remontent à l'enfance, et de la terne dynamique du couple Laura et Armando, l'histoire noue plusieurs images et notions de façon souvent paradoxale. Ainsi le Christ et les roses apparaissentils tour à tour comme la pire des tentations à laquelle il faut résister. De même, il n'est pas aisé de concilier l'horizon d'authenticité de Laura et les jeux de rôle auxquels elle se prête. Il faut sans doute suivre le parcours que ces valeurs dessinent pour envisager de comprendre l'enjeu de la « folie » de Laura, et le rapport entre codes sociaux et vérité profonde des êtres.

As long as enigmatic, the short story "A imitação da rosa", which features two female models, Laura and her friend Carlota, seems labyrinthic. Around the relationships of the two women, and the lackluster dynamic of the couple Laura and Armando, the story ties together several images and notions in often paradoxical ways. Thus Christ and the roses appear in turn as the worst temptation to be resisted. Likewise, it is not easy to reconcile Laura's demand for authenticity with the role-playing games to which she lends herself. We must undoubtedly follow the course that these values outline to understand the question of Laura's "madness", and the relationship between social codes and the deep truth of human beings.

\section{INDEX}

Mots-clés : Laços de família, A imitação da rosa, imitation, tentation, aliénation, métamorphose Keywords : Laços de família, A imitação da rosa, imitation, temptation, alienation, metamorphosis

\section{AUTEUR}

\section{MICHEL RIAUDEL}

Sorbonne Université, CRIMIC 